\title{
Fracturas en el plano coronal del húmero distal
}

\author{
J.C. Borrás Cebrián( ${ }^{(1)}$, I. Guillen ViCente ${ }^{(2)}$, M. Guillen ViCente ${ }^{(2)}$, \\ F. García de LuCAS ${ }^{(3)}$
}

(1) Médico Residente. Servicio de Cirugía Ortopédica y Traumatología. Hospital Universitario Doctor PESET. VALENCIA

(2) Médico Adjunto del Servicio de Traumatología. Clínica Cemtro. Madrid

(3) JeFE de la UNidad de Miembro Superior del Servicio de TRaumatología. CLÍNICA CEMTRO. MADRID

Correspondencia:

Dr. Juan Carlos Borrás Cebrián

Hospital Dr. Peset. Servicio de Cirugía Ortopédica y Traumatología

Av. Gaspar Aguilar, s/n

46023 Valencia. España

Telf: 961262577

E-mail: juanboce@gmail.com

\begin{abstract}
Son lesiones que comprenden las fracturas del cóndilo y/o de la tróclea humeral. Suponen el $1 \%$ de las fracturas del codo y el $6 \%$ de las fracturas del húmero distal. Las más frecuentes son las fracturas del cóndilo humeral tipo I según la clasificación de Bryan y Morrey. Analizamos 16 casos de este tipo de lesiones tratadas con reducción abierta y osteosíntesis con tornillos y valoramos los resultados funcionales. Obtuvimos la consolidación en el $100 \%$ de los casos con ausencia de dolor o dolor leve. El $87,5 \%$ de los casos presentaron una movilidad activa en flexión entre $120^{\circ}$ y $140^{\circ}$, en extensión de $-10^{\circ}$ a $-30^{\circ}$. Prono-supinación completa en 15 casos y un déficit de pronación de $60^{\circ}$ y de supinación de $50^{\circ}$ en un caso. El $100 \%$ volvieron a su actividad laboral sin restricciones en 11 semanas. En el seguimiento (11,3 meses de media, rango: 4$29), 2$ pacientes $(12,5 \%)$, necesitaron de cirugía por rigidez articular (artrolisis abierta). No hubo ningún caso de necrosis avascular. Subrayamos la importancia de obtener una reducción anatómica suficientemente estable, siendo la osteosíntesis abierta el mejor método para conseguirlo y así permitir la movilización precoz del codo y una la correcta recuperación funcional.
\end{abstract}

Palabras clave: osteosíntesis, capitellum, fractura codo, tornillos canulados.
These are injuries which comprise condyle fractures and fractures of the humeral trochlea. They constitute a $1 \%$ of elbow fractures and $6 \%$ of distal humeral fractures. The more usual fractures are those of the humeral condyle type I according to Bryan and Morrey's classification. We analyzed 16 cases of this type of injuries treated with open reduction and ostheosynthesis with threaded screws and studied the functional results during follow-up. We obtained a fixation of fracture in $100 \%$ of the cases without pain nor mild pain. $87,5 \%$ of the cases presented an active motion in flexion between $120^{\circ}$ $140^{\circ}$, in extension of $10^{\circ}-30^{\circ}$. There was complete pronosupination in 15 cases and a deficit of pronosupination of $60^{\circ}(\mathrm{P})$ and $50^{\circ}(\mathrm{S})$ in one case. All the patients decided to go back to work without restrictions for an average time of 11 weeks. During the time of follow-up (11,3 months), two patients $(12,5 \%)$ needed a secondary surgery because of stiff joint (open arthrolysis). There was no case of avascular necrosis. We infer in the importance of obtaining an anatomical reduction of enough stability, being the open ostheosynthesis the best procedure for it and allowing the earlier motion of the elbow to achieve a correct functional healing.

Key words: osteosynthesis, capitellum, elbow fracture, canulated screws.

Rev. Iberam. Cir. Mano - Vol. 36 - Núm. 1 Mayo 2008 (35-41) 


\section{INTRODUCCIÓN}

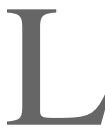

as fracturas en el plano coronal del húmero distal fueron descritas por Hahn en 1853 y por Steinthal en 1898 , tienen una baja incidencia y, afectan al cóndilo humeral (capitellum) y/o a la tróclea humeral. Pueden aparecer de forma aislada, combinadas entre sí o asociadas a otras lesiones. Las fracturas del capitellum suponen el $1 \%$ de las fracturas del codo y el $6 \%$ de las fracturas del húmero dis$\operatorname{tal}^{1,3}$. Las fracturas aisladas de la tróclea, descritas por Laugier en 1853, son más infrecuentes que las del capitellum ${ }^{4}$ y suelen aparecer asociadas a luxaciones posteriores del codo o como extensión del trazo de fractura coronal de una fractura de capitellum. Este tipo de fracturas son consecuencia del impacto de la cabeza del radio sobre el húmero distal, produciendo un mecanismo de cizallamiento, que suele ocasionar el desplazamiento de los fragmentos, a veces con conminución, dependiendo del grado de flexión del codo.

Bryan y Morrey ${ }^{5}$ clasificaron a estas fracturas en tres tipos: Tipo I (Hahn-Steinthal) co-

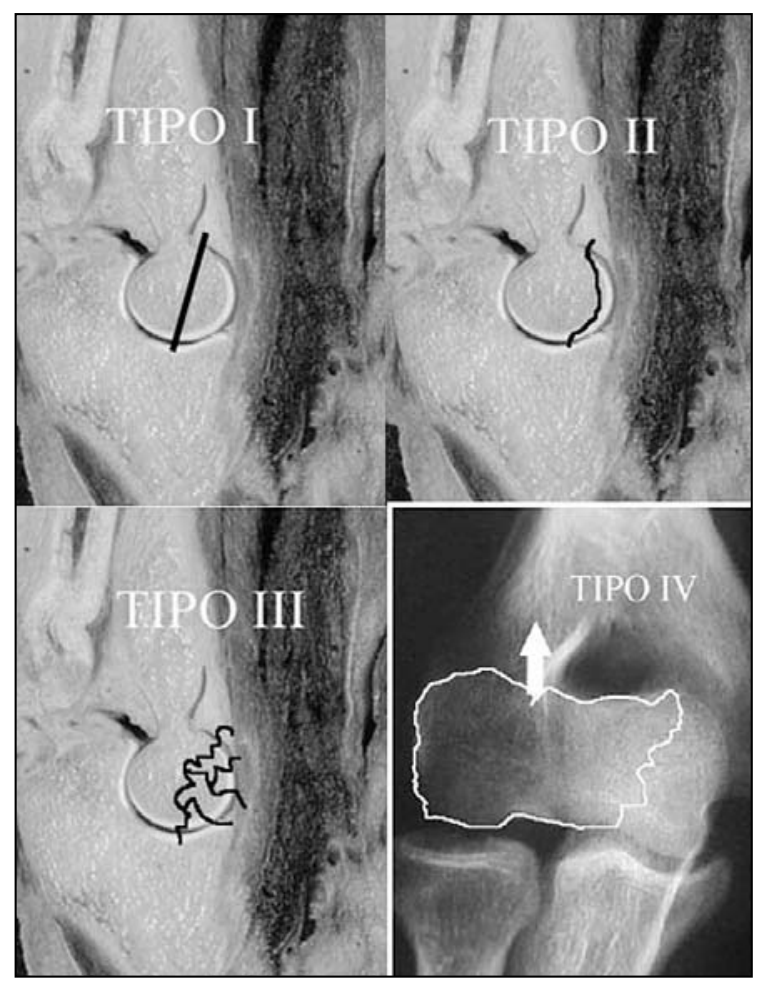

Figura 1. Clasificación de las fracturas coronales de cóndilo humeral de Bryan y Morrey, en tres tipos, a los que McKee et al. ${ }^{6}$ añadieron un cuarto. rrespondiente a un fragmento osteocondral de gran tamaño que a menudo comprende la estructura completa del capitellum; Tipo II (Kocher-Lorenz) que comprende un fragmento condral con una delgada capa de hueso subcondral, menos frecuentes que las fracturas tipo I; y Tipo III o fractura conminuta con diversos grados de desplazamiento ${ }^{1,2}$. Posteriormente McKee et al. describieron un cuarto tipo (Tipo IV) que incluía el capitellum y una porción variable de la tróclea humeral ${ }^{3,6}$ (Figura 1).

Debido a la baja incidencia de este tipo de fracturas no existen series publicadas con un gran número de casos que avalen un tratamiento idóneo para estas lesiones. Las fracturas de tipo I son las que ocasionan mayor controversia. La tendencia actual es realizar un tratamiento quirúrgico con diversos métodos de osteosíntesis ${ }^{4-16}$, aunque en el pasado algunos autores recomendaron el tratamiento con reducción cerrada ${ }^{17,18}$. Hemos analizado los resultados obtenidos tras la reducción abierta y fijación interna de las fracturas en el plano coronal del húmero distal.

\section{MATERIAL Y MÉTODO}

Entre Enero de 2000 y Enero de 2007 se trataron 17 pacientes con fracturas en el plano coronal del húmero distal en la misma institución hospitalaria, con un seguimiento medio de 11,3 meses (rango: 4-29). Se incluyeron lesiones aisladas del capitellum y/o tróclea y, asociadas a fracturas en el plano sagital. De los 17 casos, se excluyó uno por tratarse de una lesión de $c a$ pitellum aislada no desplazada que fue tratada de forma conservadora con éxito. Todos los pacientes fueron valorados clínica y radiológicamente mediante radiografías antero-posterior y lateral del codo asociando un estudio mediante tomografía axial computerizada (TAC) en los casos en los que no se obtuvieron proyecciones radiológicas adecuadas como estudio complementario (6 pacientes). El TAC puede poner de manifiesto pequeños fragmentos osteocondrales o trazos de fractura sin desplazar que no se observan en las radiografías convencionales. Las fracturas se clasificaron en base a los criterios de Bryan-Morrey y McKee et al. ${ }^{3,6}$. 


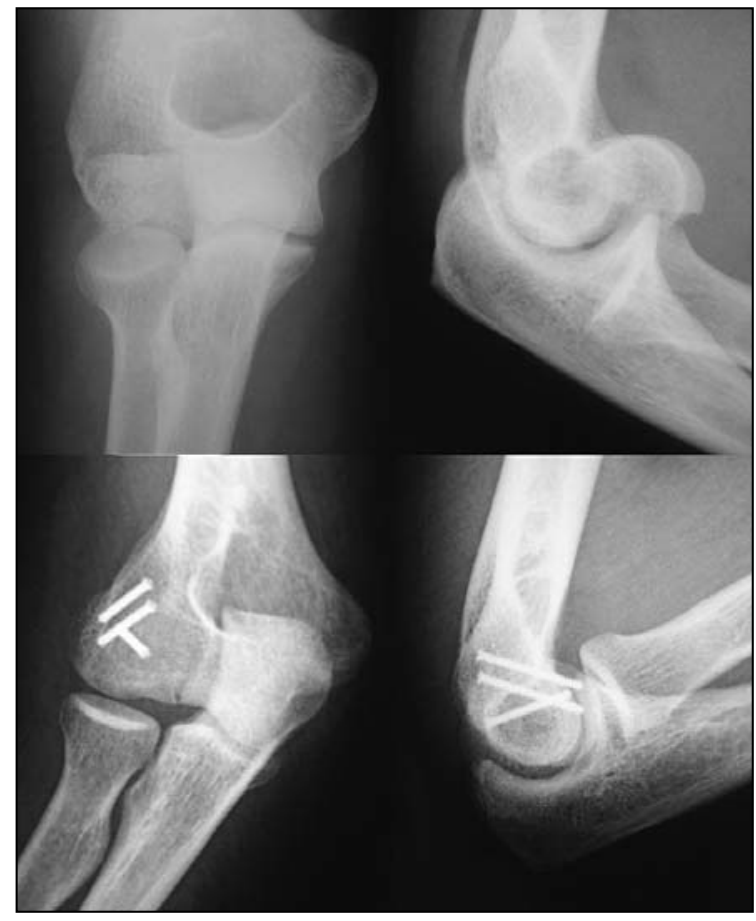

Figura 2. Fractura tipo I (Hahn-Steinthal) desplazada de cóndilo humeral. Osteosíntesis con tornillos canulados postero-anteriores.

De los dieciséis casos, nueve fueron hombres y siete mujeres. La edad media fue de 44,8 años (rango: 26-79). El lado derecho fue el afectado en 11 casos $(68,75 \%)$ y el izquierdo en 5 casos $(31,25 \%)$. Cuatro de los casos presentaron lesiones asociadas, uno con fractura de diáfisis humeral homolateral, otro con fractura de radio distal homolateral y dos con fractura de cabeza del radio homolateral. La etiología fue una caída casual en 13 casos $(81,25 \%)$, una caída desde altura en un caso $(6,25 \%)$ y accidente de tráfico en dos pacientes $(12,5 \%)$. En cuanto al tipo de fractura, seis casos $(37,5 \%)$ fueron Tipo I, uno de ellos asoció fractura de la cabeza del radio; un caso $(6,25 \%)$ fue Tipo II y; ocho casos $(50 \%)$ fueron tipo IV, uno de ellos con conminución de la tróclea que no permitió la osteosíntesis pero sí del capitellum y otro con fractura de cabeza de radio. No hubo ningún caso Tipo III. Se ha incluido en la serie un caso $(6,25 \%)$ que presentó una fractura aislada de tróclea humeral en el plano coronal.

Todos los pacientes fueron intervenidos por el mismo cirujano (FGL) y el tiempo transcurrido desde la lesión hasta la osteosíntesis fue 4,3 días de media (rango: 0-13). El abordaje

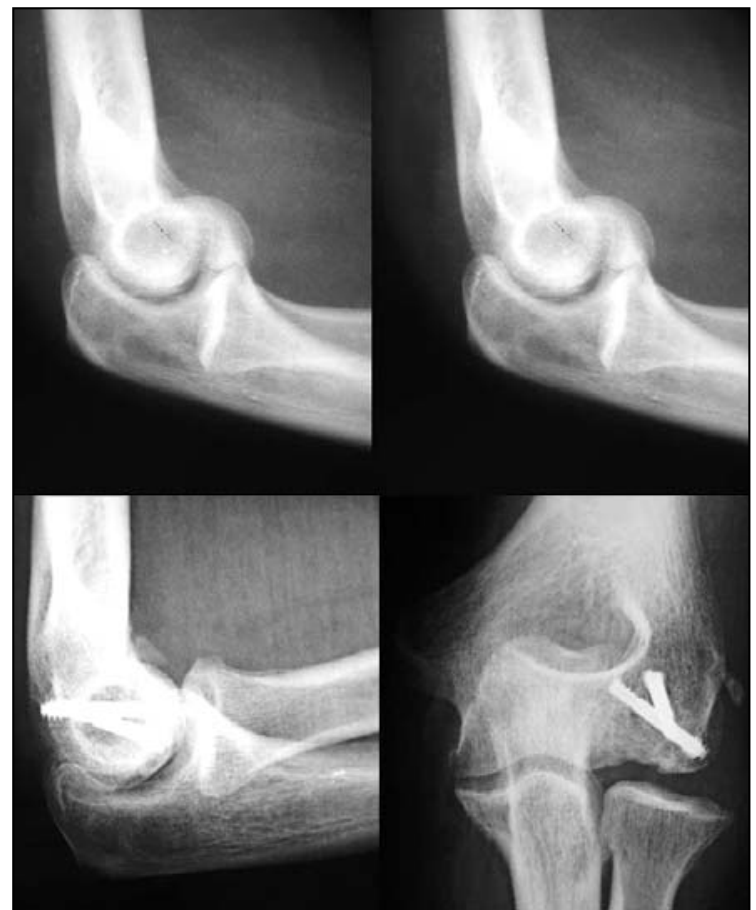

Figura 3. Fractura tipo I (Hahn-Steinthal) desplazada. Osteosíntesis antero-posterior.

lateral de Kocher se utilizó en 15 pacientes y en un caso, una vía antero-medial, por presentar fractura coronal de tróclea humeral asociada a fractura de apófisis coronoides tipo II. El método de osteosíntesis empleado de forma preferente fue el de tornillos canulados sin cabeza introducidos desde anterior a posterior (diez casos) tipo Whipple-Herbert, Herbert, Acutrak ${ }^{\circledR}$ y Miniacutrak ${ }^{\circledR}$ y tornillos a compresión de pequeños fragmentos además de los antes descritos desde posterior a anterior en seis casos (Figuras 2 y 3). Se mantuvo una inmovilización postoperatoria mediante férula braquio-antebraquial con el codo a $90^{\circ}$ y el antebrazo en posición neutra durante 14 días de media (rango: 7-30), y posteriormente se llevó a cabo un programa de rehabilitación que se prolongó 90 días (rango: 60-150). Las lesiones asociadas a las fracturas del plano coronal se trataron mediante fijación interna con placa la fractura de diáfisis humeral, con placa palmar la fractura de radio distal, con tornillos de minifragmentos una de las fracturas de cabeza de radio y, con exéresis del fragmento osteocondral la otra fractura de cabeza de radio. 


\section{RESULTADOS}

En todos los casos se obtuvo la consolidación de la fractura siendo difícil precisar el tiempo necesario para la curación debido al sistema de fijación que no permite observar callo óseo; no obstante la compresión conseguida durante la cirugía se mantuvo durante todo el seguimiento por lo que podemos concluir que en un hueso esponjoso, al cabo de 4 ó 6 semanas se acepta que la lesión ha consolidado. En los dieciséis pacientes se observó una correcta congruencia radiológica articular, tanto en el plano coronal como en el sagital y, no hubo ningún signo clínico ni radiográfico de necrosis avascular de los fragmentos.

La totalidad de los pacientes mostró ausencia de dolor o dolor leve al final del estudio. El rango de flexión activa fue de $120^{\circ}$ en once pacientes, $130^{\circ}$ en dos pacientes y $140^{\circ}$ en otro

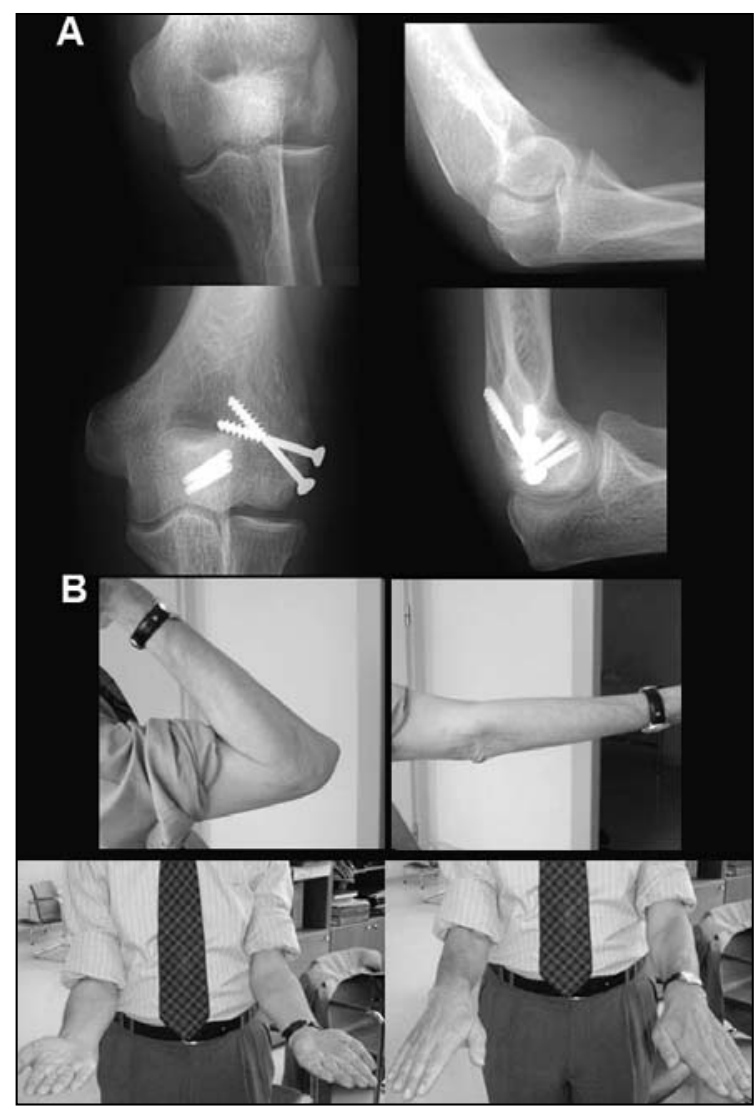

Figura 4. A. Fractura tipo IV asociada a fractura sagital del epicóndilo. Osteosíntesis con tornillos canulados Miniacutrak ${ }^{\circledR}$ del capitellum y tornillos $A O$ pequeños fragmentos en epicóndilo. B. Resultado funcional de la lesión al final del seguimiento. caso. La extensión fue de $-10^{\circ}$ en nueve pacientes, de $-20^{\circ}$ en dos pacientes y $-30^{\circ}$ en tres pacientes. El rango de pronación fue $\geq 70^{\circ}$ y el de supinación $\geq 60^{\circ}$ en quince pacientes; en un caso la pronación fue de $60^{\circ}$ y la supinación de $50^{\circ}$. Hubo dos pacientes que cursaron con rigidez en el codo y precisaron una artrolisis a los cuatro meses de la cirugía inicial; en ambos el tiempo de inmovilización se prolongó durante cuatro semanas debido a la desinserción del ligamento colateral radial durante la fijación interna. El resultado final en cuanto a dolor y movilidad, fue muy satisfactorio, comparable a los resultados del resto de casos (ambos casos flexión $110^{\circ}$, extensión $-20^{\circ}$, prono-supinación completa) (Figura 4). Un paciente que presentaba molestias durante la flexoextensión del codo debido a la protrusión articular del tornillo introducido desde posterior a anterior, precisó la retirada del implante al cabo de 6 semanas.

Todos los pacientes se incorporaron a su actividad laboral previa en un tiempo medio de 11 semanas (rango: 3-19), dependiendo del tipo de trabajo que realizasen. Los dos casos de artrolisis también se incorporaron a su actividad pero tras un período de tiempo más prolongado.

\section{DISCUSIÓN}

Las fracturas articulares de trazo coronal del húmero distal son muy poco frecuentes, siendo difícil encontrar series con elevado número de $\operatorname{casos}^{7,10,16,19}$. El mecanismo de lesión de estas fracturas implica un impacto de la cabeza del radio con el capitellum y/o tróclea que producirá un fractura por cizallamiento o una fractura impactada dependiendo de la flexión del codo en el momento del traumatismo, más que de la intensidad de la fuerza lesional, como lo demuestra el hecho de que en nuestra serie la mayoría de las fracturas se produjeron tras una caída casual.

La incidencia superior de estas fracturas en el género femenino contrasta con la proporción ligeramente mayor a favor de los hombres en nuestra serie, nueve hombres frente a siete mujeres. Posiblemente por tratarse de una serie de pacientes de Mutua Laboral. 


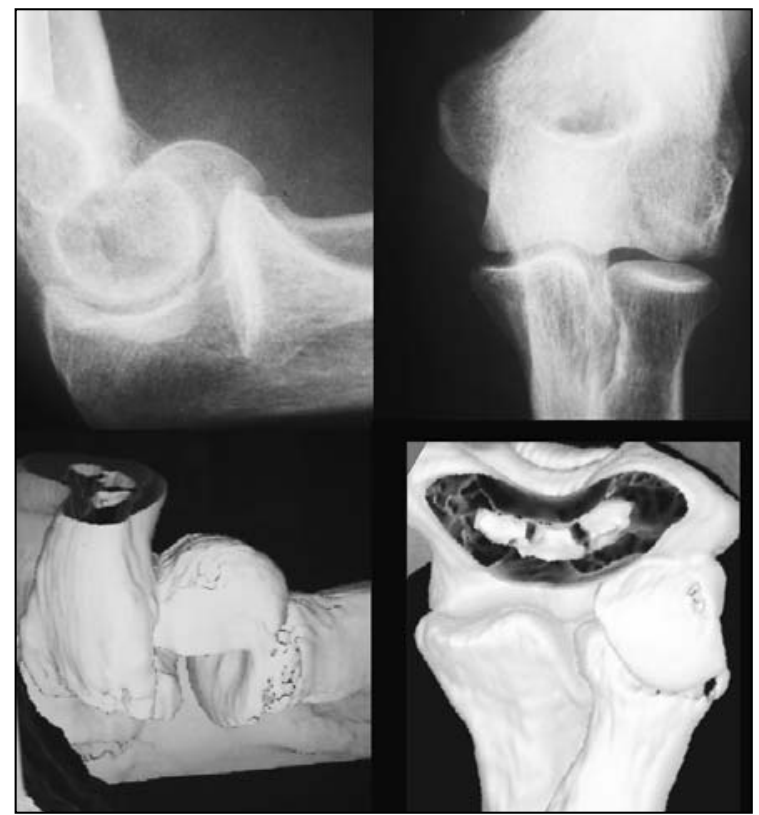

Figura 5. Comparación de imagen de fractura Tipo I en la radiología simple en el TAC.

Es controvertido extraer conclusiones acerca del tratamiento de este tipo de fracturas o determinar cual es el más adecuado, ya que la experiencia que se puede acumular de forma individual es escasa. Son lesiones de difícil diagnóstico sobre todo los casos Tipo II en los que debido al pequeño tamaño del hueso subcondral del fragmento puede pasar desapercibido con el estudio radiológico convencional. La proyección lateral correctamente realizada permite el diagnóstico en la mayoría de las fracturas como lo demuestra nuestra serie en la que tan solo fue necesaria la realización de TAC en 6 casos.

Basándose en los principios básicos del tratamiento de las fracturas articulares, parece evidente que el mantener la estructura articular en una situación anatómica es fundamental para restablecer de forma precoz la función de dicha articulación. En el caso de las fracturas desplazadas, la reducción abierta y la osteosíntesis son el único método que cumple estos objetivos. Así pues, el tratamiento quirúrgico ofrece resultados funcionales más satisfactorios si se consigue una fijación estable que permita una rápida movilización. En el caso de no poder fijar pequeños fragmentos osteocondrales es preferible la exéresis de éstos y un tratamiento rehabilitador precoz.

Existe controversia en cuanto al mejor método de fijación interna; para algunos autores es preferible la osteosíntesis desde anterior a posterior $^{16}$ y para otros, desde posterior a anterior ${ }^{20}$. En nuestra serie, se han empleado mayoritariamente tornillos canulados, al igual que en otras publicadas ${ }^{7,21}$ sin cabeza desde anterior a posterior y creemos que esta técnica resulta más cómoda pues evita la disección de la parte posterior de la paleta humeral y no hemos encontrado diferencias en cuanto a los resultados obtenidos (Tabla I). El defecto condral que deja la introducción del tornillo es pequeño y no tiene repercusión funcional siempre que quede oculto en el hueso subcondral. El abordaje lateral de Kocher permite en la mayoría de los casos la reducción de los fragmentos; si existe una fractura asociada de la tróclea o una fractura aislada de ésta, es necesario desinsertar a nivel proximal el ligamento colateral radial para acceder a esta zona más medial de la paleta humeral o recurrir a una vía medial o antero-medial.

En el caso de existir una fractura de cabeza de radio asociada, se debe intentar la reducción y fijación, y si no es posible, la exéresis del fragmento inestable o de toda la cabeza lo que no modifica la pauta de rehabilitación precoz de las fracturas en el plano coronal de la paleta humeral.

La evaluación radiológica de la consolidación de estas fracturas resulta difícil pues no existe callo, no obstante la ausencia de líneas de radiolucencia en el trazo de fractura y/o de aumento de densidad en los fragmentos constituyen elementos que avalan la curación ósea y la ausencia de necrosis ósea avascular.

Como en cualquier otra lesión articular, la rehabilitación precoz constituye un pilar fundamental para obtener un buen resultado funcional. En las ocasiones en las que por uno u otro motivo el tiempo de inmovilización se prolonga, la aparición de una rigidez articular es más probable. 


\section{Tabla I - REVISIÓN COMPARATIVA DE CASOS DE FRACTURAS CORONALES DEL HÚMERO DISTAL}

\begin{tabular}{|c|c|c|c|c|}
\hline \multicolumn{5}{|c|}{ Revisión comparativa } \\
\hline Serie & Año & Casos & Tratamiento & Resultados \\
\hline Poynton et al. ${ }^{11}$ & 1998 & 12 & $\begin{array}{l}\text { - } 6 \text { casos con agujas de } \\
\text { Kirschner y } 6 \text { semanas de } \\
\text { inmovilización } \\
-6 \text { casos con tornillos de } \\
\text { Herbert postero-anteriores y } \\
\text { movilidad inmediata }\end{array}$ & $\begin{array}{l}\text { - } 1 \text { caso infección de aguja } \\
\text { con déficit funcional. } \\
\text { - } 11 \text { casos de buenos } \\
\text { resultados funcionales. } \\
\text { - Tiempo de reincorporación } \\
\text { a las actividades previas: } \\
\text { Herbert: } 5,5 \text { semanas } \\
\text { Kirschner: } 9 \text { semanas }\end{array}$ \\
\hline De Boeck et al. ${ }^{20}$ & 2000 & $\begin{array}{l}6 \\
\text { Niños } \\
\text { entre } \\
11 \text { y } 15 \\
\text { años }\end{array}$ & $\begin{array}{l}\text { Fracturas Tipo } 1^{1,2} \text {. } \\
\text { Tornillo de pequeños } \\
\text { fragmentos postero- } \\
\text { anteriores. } 3 \text { semanas de } \\
\text { inmovilización }\end{array}$ & $\begin{array}{l}\text { - Buenos resultados } \\
\text { funcionales en todos los } \\
\text { casos. } \\
\text { - No infección, no necrosis, no } \\
\text { deformidad varo-valgo. } \\
\text { - } 2 \text { casos pérdida de flexión } \\
\text { de } 5^{\circ}-10^{\circ} \text {. }\end{array}$ \\
\hline Clough et al..$^{8}$ & 2001 & 4 & $\begin{array}{l}\text { Placa en Y apoyada sobre la } \\
\text { columna lateral, no } \\
\text { compresión de la fractura, } \\
1 \text { semana de inmovilización }\end{array}$ & $\begin{array}{l}\text { - No alteración funcional. } \\
\text { - Déficit de extensión de } 6^{\circ} \text { y } \\
\text { de } 10^{\circ} \text { de flexión. } \\
\text { - No infecciones, no necrosis } \\
\text { avascular. } \\
\text { - La ventaja es una menor } \\
\text { disección quirúrgica que con } \\
\text { tornillos tipo Herbert. }\end{array}$ \\
\hline Schindler et al. ${ }^{19}$ & 2003 & $\begin{array}{c}1 \\
\text { bilateral }\end{array}$ & $\begin{array}{l}\text {-codo dcho, Tipo I: Herbert - } \\
\text { codo izdo, Tipo I y fractura } \\
\text { sagital: Herbert y tornillos a } \\
\text { compresión. }\end{array}$ & $\begin{array}{l}\text { - Rango articular funcional. } \\
\text { - Sin dolor } \\
\text { - No limitación de actividad } \\
\text { diaria. }\end{array}$ \\
\hline Ring et al. ${ }^{7}$ & 2003 & 21 & $\begin{array}{l}\text { Fracturas en el plano coronal } \\
\text { aisladas o combinadas con } \\
\text { fracturas en el plano sagital. } \\
\text { Aconsejan la osteosíntesis } \\
\text { con tornillos canulados }\end{array}$ & $\begin{array}{l}\text { - No inestabilidad. } \\
\text { - } 6 \text { casos de rigidez. } \\
\text { - } 2 \text { neuropatías cubitales. } \\
\text { - } 1 \text { extracción de material. } \\
\text { - Rango articular de } 96^{\circ} \text {. } \\
\text { - Buenos o muy buenos } \\
\text { resultados en } 16 \text { casos. }\end{array}$ \\
\hline Sano et al. ${ }^{16}$ & 2005 & 6 & $\begin{array}{l}\text { Tornillos de Herbert postero- } \\
\text { anteriores y antero- } \\
\text { posteriores. }\end{array}$ & $\begin{array}{l}\text { - Rango articular funcional. } \\
\text { - No infección ni necrosis } \\
\text { - No neuropatía cubital } \\
\text { - No dolor }\end{array}$ \\
\hline Borrás et al. & 2008 & 16 & $\begin{array}{l}\text { Tornillos canulados sin } \\
\text { cabeza postero-anteriores y } \\
\text { antero-posteriores. } \\
\text { Osteosíntesis de lesiones } \\
\text { asociadas. }\end{array}$ & $\begin{array}{l}\text { - Rango articular funcional en } \\
14 \text { de } 16 \text { casos. No dolor } \\
\text { - } 2 \text { casos de rigidez de codo } \\
\text { que precisaron artrolisis } \\
\text { abierta. } \\
\text { - un caso de protrusión } \\
\text { articular de la osteosíntesis }\end{array}$ \\
\hline
\end{tabular}




\section{BIBLIOGRAFÍA}

1. Robinson CM. Fractures of the distal humerus. En: Bulcholz RW, Heckman JD, Courtbrown C (Eds): Rockwood and Green's. Fractures in adults. $6^{\text {th }}$ ed. Philadelphia: Lippincott, Williams \& Wilkins; 2006: 1101-7.

2. Jupiter JB, Morrey BF. Fracturas del húmero distal en adultos. En: Morrey BF. Traumatología del codo. $3^{\mathrm{a}}$ ed. Madrid; Marban; 2004: 318-21.

3. Barei DP, Hanel DP. Fracturas distales del húmero. En: Green DP, Hotchkiss RN, Pederson WC et al. Green's. Cirugía de la mano. $5^{\mathrm{a}}$ ed. Madrid: Marban; 2007: 809-43.

4. Mehdian H, McKee MD. Fractures of capitellum and trochlea. Orthop Clin North Am, 2000; 31: 115-27.

5. Bryan RS, Morrey BF. Fractures of the distal humerus. En: Morrey BF (Ed). The Elbow and its Disorders. Philadelphia, Pa: WB Saunders; 1985: 302-39.

6. McKee MD, Júpiter JB, Bamberger $\mathrm{H}$. Coronal shear fractures of the distal end of the humerus. J Bone Joint Surg Am, 1996; 78: 49-54.

7. Ring D, Jupiter JB, Gulotta L. Articular fractures of the distal part of the humerus. J Bone Joint Surg Am, 2003; 85: 232-8.

8. Clough TM, Jago ER, Sidhu DPS, et al. Fractures of the capitellum: a new method of fixation using a maxillofacial plate. Clin Orthop, 2001; 384: 232-6.

9. Elkowitz SJ, Kubiak EN, Polatsch $\mathrm{D}$, et al. Comparison of two headless screw designs for fixation of capitellum fractures. Bull Hosp Jt Dis, 2003; 61: 123-6.

10. Elkowitz SJ, Polatsch DB, Kenneth $A$, et al. Capitellum fractures: a biomechanical evaluation of three fixation methods. J Orthop Trauma, 2002; 16: 503-6.

11. Poynton R, Kelly IP, O'Rourke SK. Fractures of the capitellum, a comparison of two fixation methods. Injury, 1998; 29: 341-3.

12. Sen MK, Sama L, Helfet DL, et al. Open reduction and internal fixation of coronal fractures of the capitellum. J Hand Surg Am, 2007; 32: 1462-5.

13. Mighell MA, Harkins D, Klein D, et al. Technique for internal fixation of capitellum and lateral trochlea fractures. J Orthop Trauma, 2006; 20: 699-704.
14. Mahirogullari M, Kiral A, Solakoglu C, et al. Treatment of fractures of the humeral capitellum using Herbert screws. J Hand Surg Br, 2006; 31: 320-5.

15. Dubberley JH, Faber HJ, Macdermid JC, et al. Outcome after open reduction and internal fixation of capitellar and trochlear fractures. J Bone Joint Surg Am, 2006; 88: 46-54.

16. Sano S, Rokkaku T, Saito S, et al. Herbert screw fixation of capitellar fractures. J Shoulder Elbow Surg, 2005; 14: 307-11.

17. Alvarez E, Patel MR, Nimberg $\mathrm{G}$, et al. Fracture of the capitullum humeri. J Bone Joint Surg Am, 1975; 57: 1093-6.

18. Ochner RS, Bloom H. Closed reduction of coronal fractures of the capitellum. J Trauma, 1996; 40: 199-203.

19. Schindler OS. Bilateral capitellum humeri fracture. A case report and review of the literature. J Orthop Surg, 2003; 11: 207-212.

20. De Boeck H, Pouliart N. Fractures of the capitellum humeri in adolescents. Int Orthop, 2000; 24: 246-8.

21. Cheung EV. Fractures of the capitellum. Hand Clin, 2007; 23: 481-6. 